\title{
Control of Metabolite Secretion in Bacillus subtilis
}

\author{
By E. L. SPECK* AND E. FREESE \\ National Institute of Neurological Diseases and Stroke, National Institutes of \\ Health, Public Health Service, U.S. Department of Health, Education \\ and Welfare, Bethesda, Maryland, 20014, U.S.A.
}

(Received I6 January 1973; revised 22 March 1973)

SUMMARY

Bacillus subtilis, grown in media containing amino acids and glucose or other carbohydrates (including glycerol), secreted pyruvate, acetate, acetoin, butanediol, and small quantities of isobutyrate and isovalerate. The enzymes producing acetoin and butanediol were specifically induced by acetate and weakly by propionate. Even when these enzymes were present, acetate accelerated the rate of acetoin production. Since acetoin and butanediol are readily interconverted (via butanediol dehydrogenase), they serve as an oxidation-reduction buffer for NAD/NADH. When carbohydrate was exhausted, acetate and, more slowly, acetoin were oxidized.

Mutants unable to produce acetoin or acetate were blocked in the metabolic path from the carbohydrate to these compounds. Other mutants unable to re-utilize the compounds were either deficient in the necessary enzymes, e.g. of citric acid cycle, or they stopped growth so early that they could not use up the carbohydrate. In particular, a uracil-requiring mutant stopped growth precociously, did not oxidize acids or acetoin, and was unable to sporulate. Addition of uracil restored all these properties to normal. The differences in secretion and re-utilization of metabolites can be employed to classify unidentified sporulation mutants.

\section{INTRODUCTION}

Bacteria secrete specific metabolites that reflect their enzyme composition and their growth conditions. In a rich medium containing carbohydrates such as glucose (Glc), Enterobacter or certain species of Bacillus typically produce acetoin, a phenomenon which helps to distinguish these organisms from others (Smith, Gordon \& Clark, 1952). The secretion usually increases toward the end of growth and some of the released compounds can later be reutilized (Kominek \& Halvorson, 1965). The control of secretion may be important not only for growth on (or fermentation of) certain substrates, but also at the end of growth for the maintenance of bacterial integrity or for the development of dormant forms such as spores. Mutations preventing the accumulation or re-utilization of certain compounds can therefore prevent the normal developmental processes.

In this paper the metabolites secreted and re-utilized by Bacillus subtilis are analysed and the mechanisms controlling their production are examined. Acetate, produced during growth, induces the enzymes that cause the accumulation of acetoin and butanediol. Mutants can be classified according to their inability to produce or metabolize acids or acetoin. Most mutants lacking any one of these properties are cacogenic, i.e. they cannot sporulate normally. This can be employed to characterize the biochemical deficiency of cacogenic mutants as is shown for mutants blocked in known biochemical reactions.

* Present address: Department of Infectious Diseases, Strong Memorial Hospital, Rochester, New York I4620, U.S.A. 


\section{METHODS}

Bacteria. The transformable and sporulating Bacillus subtilis strain 60015, requiring L-tryptophan (Trp) and L-methionine (Met), was used as the standard strain. Various mutants had previously been derived either from this strain or from other transformable strains originating in Spizizen's strain I68. For strain 6г 3 Iо see Freese, Klofat \& Galliers (1970), for 6I I I I see Freese, Oh, Freese, Diesterhaft \& Prasad (1972), for 6I I04 and 6r 437 see Diesterhaft \& Freese (1973). Strain 600I8, requiring L-histidine, uracil and Trp, was obtained from Nester (his strain SB 5). Strains 6I437, requiring Trp and deficient in pyruvate carboxylase activity (strain C 50), and 6I460, requiring Trp and deficient in citrate synthase (strain SB 59), were obtained from Hoch.

Media. TBAB plates contained $33 \mathrm{~g} / \mathrm{l}$ Difco Tryptose Blood Agar Base. Nutrient sporulation medium with (NSMP) and without (NSM) potassium phosphate, minimal salts medium $\mathrm{N}$ and the citrate-containing medium $\mathrm{M}$ (Freese et al. 1972), were supplemented with to $\mu \mathrm{g} \mathrm{Met} / \mathrm{ml}$ and $25 \mu \mathrm{g}$ Trp/ml, whereas the Trp-limiting casein hydrolysate medium contained $5 \mathrm{~g}$ Glc, $8 \mathrm{~g}$ vitamin-free casein hydrolysate (Nutritional Biochemicals Corp.), Io $\mathrm{mg} \mathrm{Met}$, and only I mg Trp/l of $\mathrm{N}$ medium.

Growth conditions. Bacteria were grown as a lawn on TBAB plates for $\mathrm{I} 8 \mathrm{~h}$, harvested with medium and dispersed on a Vortex Mixer. Erlenmeyer flasks or test tubes with $25 \mathrm{~mm}$ diam. were inoculated at an absorbency at $600 \mathrm{~nm}\left(A_{600}\right)$ of about 0.I (measured against medium) and shaken at $\mathrm{I} 20$ strokes/min in a reciprocal water-bath shaker at $37^{\circ} \mathrm{C}$. To ensure optimal aeration, the media occupied no more than $10 \%$ of the volume of each flask.

Determination of compounds. 'After removal of the bacteria by centrifugation, acetoin was determined chemically and pyruvate enzymatically as described previously (Freese \& Fortnagel, 1969). The colorimetric method used for acetoin determination does not distinguish between acetoin (acetylmethylcarbinol), diacetyl, diacetylmethylcarbinol and acetylbutanediol (Juni \& Heym, 1956). However, gas chromatography separated these compounds and verified that acetoin and butanediol were the major secretion products.

For gas chromatography, a I.8 m coiled glass column (4 mm inside diam.) was filled with $30 \%$ Rheoplex 400 on Chromosorb W (Analabs, Inc., North Haven, Connecticut, U.S.A.). The column temperature was $140{ }^{\circ} \mathrm{C}$. A flame ionization electrometer was used for the detection of most compounds except for lactate, which was sensed by an electron capture detector. The peaks were identified by running authentic compounds both separately and together with the sample. Ten mM-pentanedione (acetylacetone) was used as internal standard. Most of the acetoin (but not the acetate) was removed by shaking the neutral sample with ether, exposure to $0.8 \mathrm{~N}-\mathrm{KOH}$ at $37^{\circ} \mathrm{C}$ for $3 \mathrm{~h}$, or treatment with equal amounts of acetic anhydride and pyridine. Upon acidification of the sample by $\mathrm{HCl}$, acetate and the other acids were extractable by ether. The elution times of many compounds were determined (Fig. I). They were reproducible and allowed the clear identification of all major peaks observed in the bacterial supernatant. Commercial butanediol gave two peaks, one corresponding to the meso- and the other to the D,L-compounds. For quantitative determination of metabolites secreted during different periods of growth in NSM, the bacteria were filtered out, pentanedione was added (Io mM final) and $5 \mu \mathrm{l}$ of this medium were injected into the column. The observed peaks were much larger than the small peaks obtained when water or NSM were injected as controls.

Glucose was determined by the glucose oxidase method using the Glucostat reagent (Worthington Biochemical Corp., Freehold, New Jersey, U.S.A.). One vial each of the glucose oxidase and the chromogen reagent were dissolved in $95 \mathrm{ml}$ distilled water. A I.9 ml 


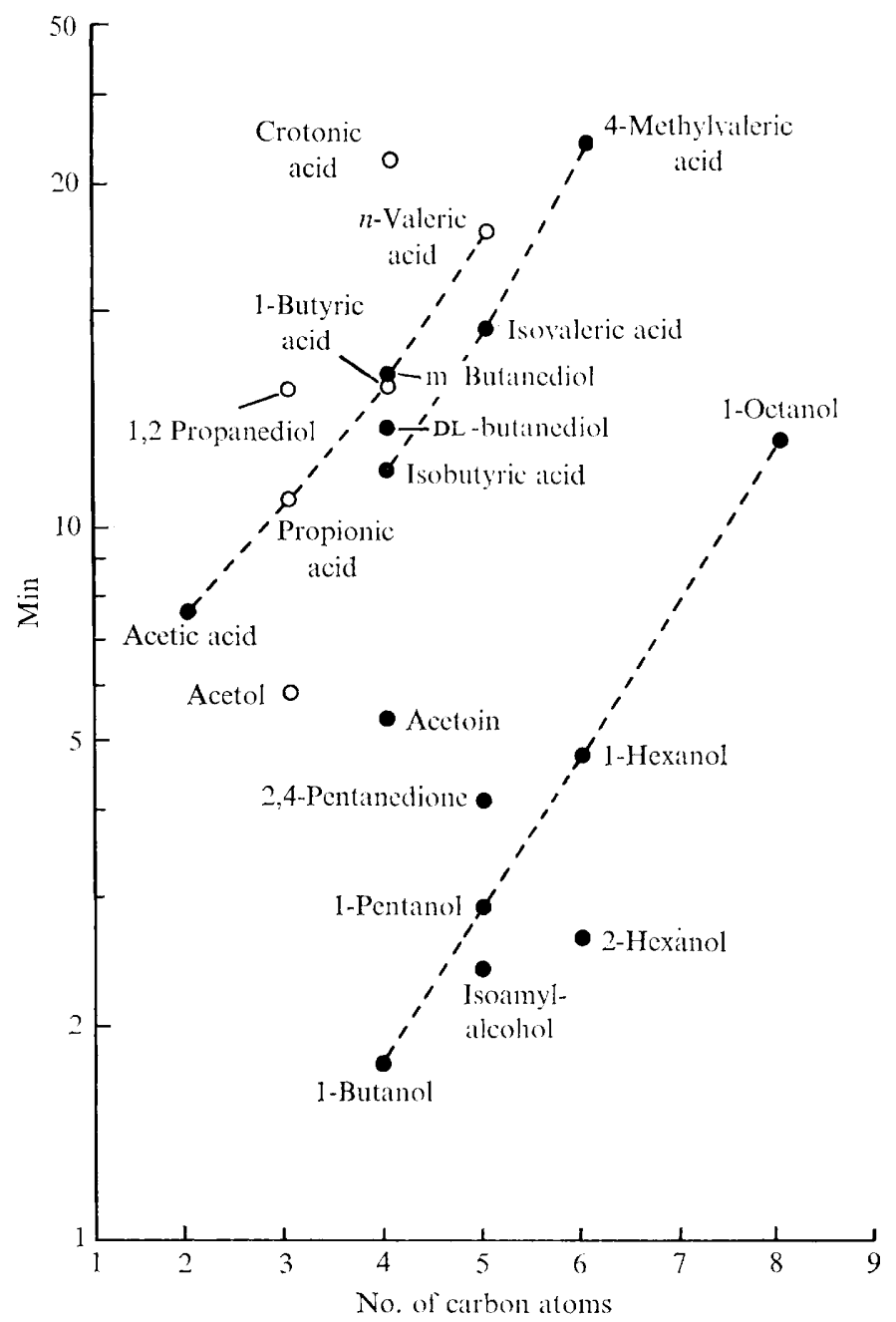

Fig. I. Gas chromatographic elution times of different compounds. The compounds (about 50 nmoles) were injected in solution in ether. Conditions are described in Methods.

sample of this glucostat mixture and $0 . \mathrm{I} \mathrm{ml}$ of the glucose solution (containing 100 to $500 \mu \mathrm{g}$ glucose $/ \mathrm{ml}$ ) were mixed and incubated for $\mathrm{I} 0 \mathrm{~min}$ at $37^{\circ} \mathrm{C}$. Ten $\mu \mathrm{l}$ of $5 \mathrm{M}-\mathrm{HCl}$ were added and the $A_{400}$ was read. Standard solutions of glucose were used for calibration.

Preparation of bacterial extracts. Bacteria were harvested in centrifuge bottles containing chloramphenicol (to give $100 \mu \mathrm{g} / \mathrm{ml}$ ), centrifuged at $\mathrm{I} 2000 \mathrm{~g}$ for $10 \mathrm{~min}$ and washed three times with $0.05 \mathrm{M}$-tris-hydroxymethylaminomethane- $\mathrm{Cl}$ (tris) at $\mathrm{pH} 8.0$.

The pellet was suspended in $0.05 \mathrm{M}$-tris, $\mathrm{pH} 8$, to give an $A_{600}$ of about 50 ; after homogenization, the bacteria were disrupted in the presence of $2 \mu \mathrm{g}$ DNase $/ \mathrm{ml}$ either by $100 \mu \mathrm{g}$ lysozyme $/ \mathrm{ml}$ at $37^{\circ} \mathrm{C}$ or by passage through a chilled French pressure cell. The membraneassociated NADH oxidation activity was lower in extracts obtained by the lysozyme method, but it could be kept reasonably low also in the other disruption method by centrifugation for $20 \mathrm{~min}$ at $45000 \mathrm{~g}$. The supernatant extract was immediately used for enzyme assays. Enzyme activities in extracts produced by the two methods were not significantly different. 

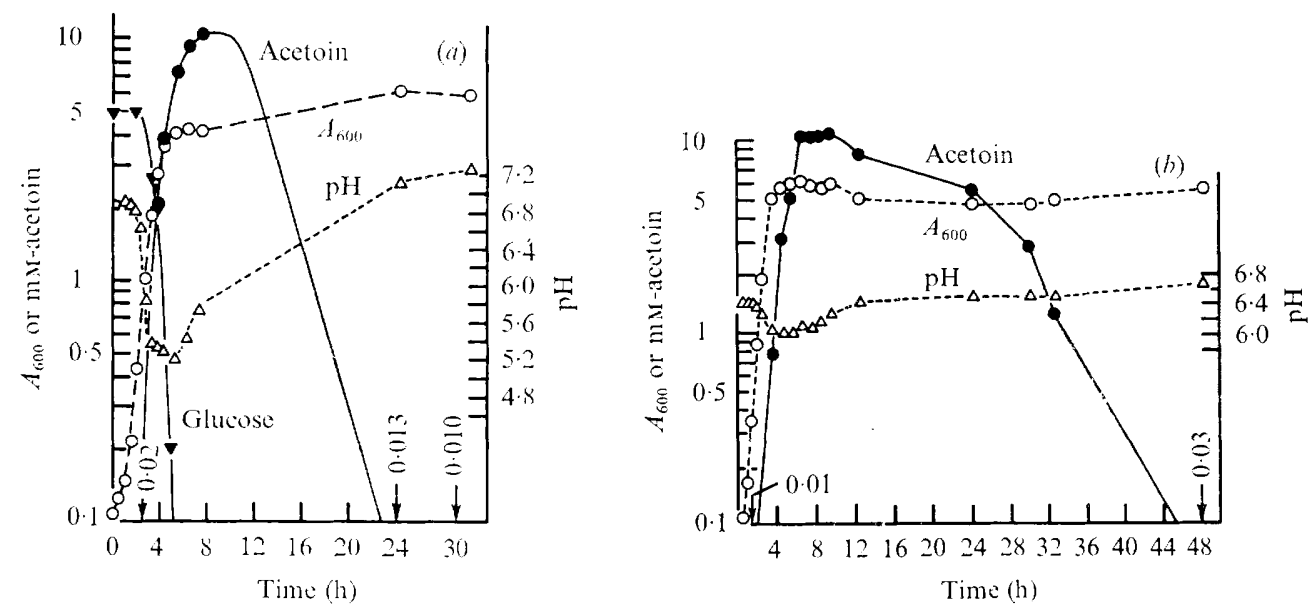

Fig. 2. $\mathrm{pH}$ changes and acetoin production in nutrient sporulation medium containing glucose (Glc). The standard strain (60015) of Bacillus subtilis was grown in: (a) NSM+0.5\% Glc; (b) NSMP $+0.5 \%$ Glc.

Assay of Protein. Protein was determined by the method of Lowry, Rosebrough, Farr \& Randall (I95I).

Enzyme assays. The acetoin-producing enzymes were first assayed by a method similar to that of Bauerle, Freundlich, Størmer \& Umbarger (I964). The assay mixture (I ml) contained $50 \mathrm{~mm}$-sodium pyruvate, I mM-thiamine pyrophosphate, I00 mM-potassium phosphate (pH 6), Iо $\mathrm{mM}-\mathrm{MgCl}_{2}$, and bacterial extract. After incubation at $37^{\circ} \mathrm{C}$ for $10 \mathrm{~min}$, the reaction was stopped by addition of $50 \mu 1 \mathrm{ION}-\mathrm{H}_{2} \mathrm{SO}_{4}$. To convert all acetolactate to acetoin, the acidified mixture was autoclaved for $5 \mathrm{~min}$ at $10 \mathrm{lb} / \mathrm{in}^{2}(\mathrm{I} \cdot 7 \mathrm{~atm})$ and then assayed for acetoin. This 'autoclaved' fraction measures the acetolactate synthase activity. The 'unautoclaved' fraction determines the overall reaction from pyruvate to acetoin and is a measure of acetolactate decarboxylase activity. Later the method of Malthe-Sørenssen \& Størmer (1970) was used for acetolactate synthase and that of Løken \& Størmer (1970) for acetolactate decarboxylase. Acetolactate (mixed with an equal concentration of acetate and ethanol) was prepared according to Krampitz (I948). The substrate was a gift of Dr H. E. Umbarger, Purdue University, U.S.A.

2,3-Butanediol dehydrogenase was assayed (at $25^{\circ} \mathrm{C}$ ) by the oxidation of reduced nicotinamide adenine dinucleotide (NADH) measured as decrease in absorbency at $340 \mathrm{~nm}$. The small NADH oxidation activity was subtracted. The assay mixture ( $\mathrm{ml}$ ) contained: Ioo mM-tris (pH 8), o.I 5 mM-NADH, 5 mM-acetoin and bacterial extract.

\section{RESULTS}

Secretion of low molecular weight metabolites by the standard strain. Our standard strain (600I5) of Bacillus subtilis, grown in nutrient sporulation medium plus glucose (Glc) either without (NSM) or with (NSMP) K-phosphate buffer, secreted several metabolites $(0.5 \%$ glucose was used in most experiments, because this concentration was best for characterization of mutants; exceptions are specifically stated). The decrease of $\mathrm{pH}$ during growth in unbuffered NSM + Glc indicated the production of acids; when all the glucose had been used up, the $\mathrm{pH}$ increased again, reflecting the further oxidation of the acids and the pro- 


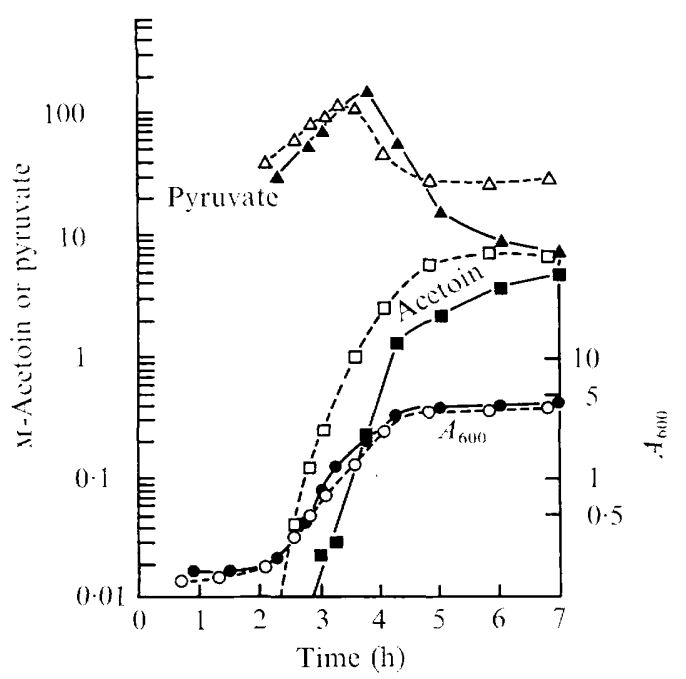

Fig. 3

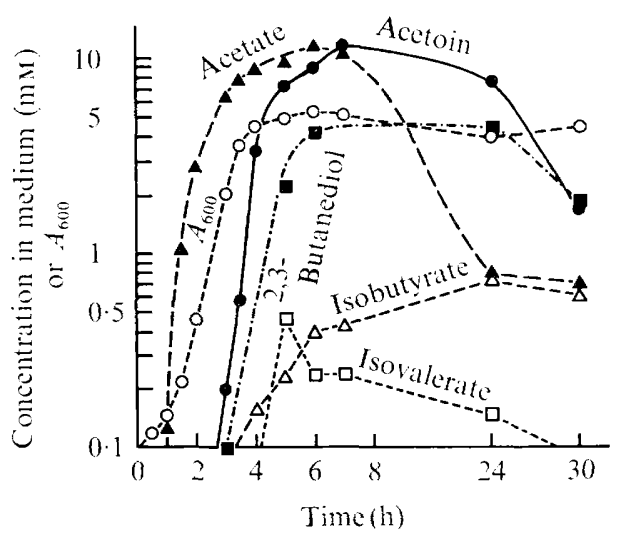

Fig. 4

Fig. 3. Production of pyruvate and acetoin by strain 60015 . Solid symbols: NSMP $+0.5 \%$ Glc. Open symbols: same + Io mM-K-acetate.

Fig. 4. Production of different secretion products, as determined by gas chromatography. Strain 60015 was grown in NSM + $1 \%$ Glc. At different times samples were withdrawn, 2,4-pentanedione was added as internal standard to give $10 \mathrm{~mm}$, and $5 \mu \mathrm{l}$ samples were directly injected into the gas chromatograph. The peaks were quantitated with respect to the pentanedione peak and their quantities (in nmoles) were read off calibration curves determined for each compound in NSM medium.

duction of free amino compounds (Fig. $2 a$ ). The major acids secreted into the medium were pyruvate, measured enzymatically (Fig. 3), and acetate, measured by gas chromatography (Fig. 4). Smaller concentrations of isobutyrate and isovalerate were also observed (Fig. 4). Compared to pyruvate, lactate was produced only in very small quantities (qualitatively detected by gas chromatography, using an electron capture detector), in agreement with the virtual absence of lactic dehydrogenase activity in well aerated B. subtilis (Yoshida \& Freese, 1965). Citric acid cycle intermediates could not be detected by the methods used.

Following the production of pyruvate and acetate, the concentrations of acetoin and then 2,3-butanediol increased in the medium (Fig. 4). Essentially the same amount of acetoin was produced in the unbuffered NSM (Fig. $2 a$ ) and the phosphate buffered NSMP (Fig. $2 b$ ) media, irrespective of the extent of the $\mathrm{pH}$ decrease. Acetoin was produced in similar quantities in NSM plus $0.5 \%$ of fructose, glucose, mannose or glycerol, and at much smaller concentrations from gluconate or ribose; L-malate (which is an excellent carbon source for growth), citrate, or pyruvate (which is taken up very poorly) effected no more acetoin production than NSM alone (Table I). Apparently, intracellular pyruvate accumulated in sufficient concentration only from carbohydrates that produce PEP. 2,3-Butanediol gave rise to two gas chromatographic peaks, which must correspond to the meso- and either the L- or the D-forms (or both). No significant gas chromatographic peak of diacetyl was seen (it would elute very early). The sequential production of pyruvate, acetoin, and butanediol agrees with the sequence of biochemical reactions (Fig. 5).

The reduction of acetoin was greatly enhanced by anaerobic conditions (Fig. 6). In this experiment bacteria were grown in synthetic medium (MG) containing acetoin. The concentration of acetoin remained constant so long as aeration was maintained, but it decreased, 


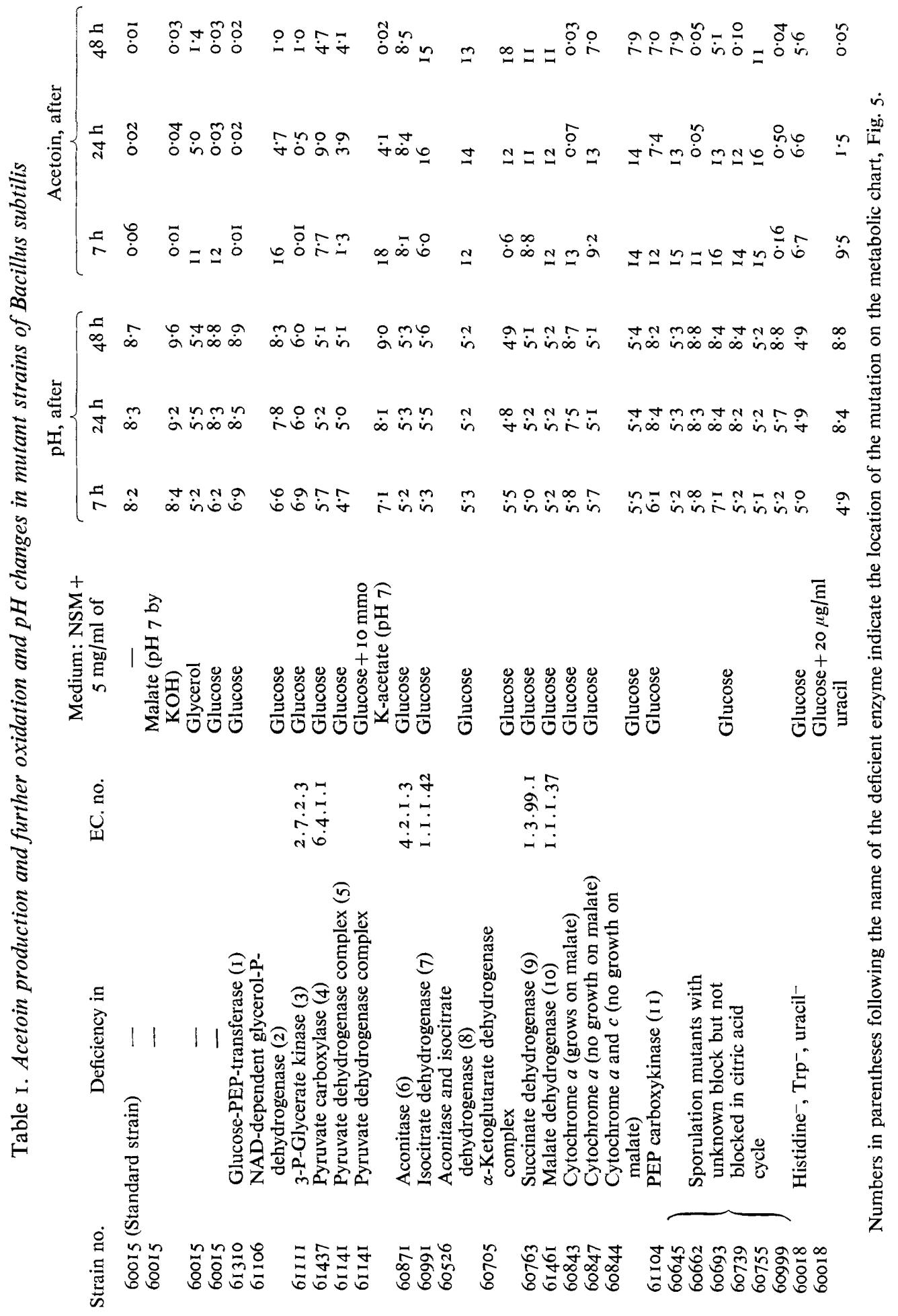




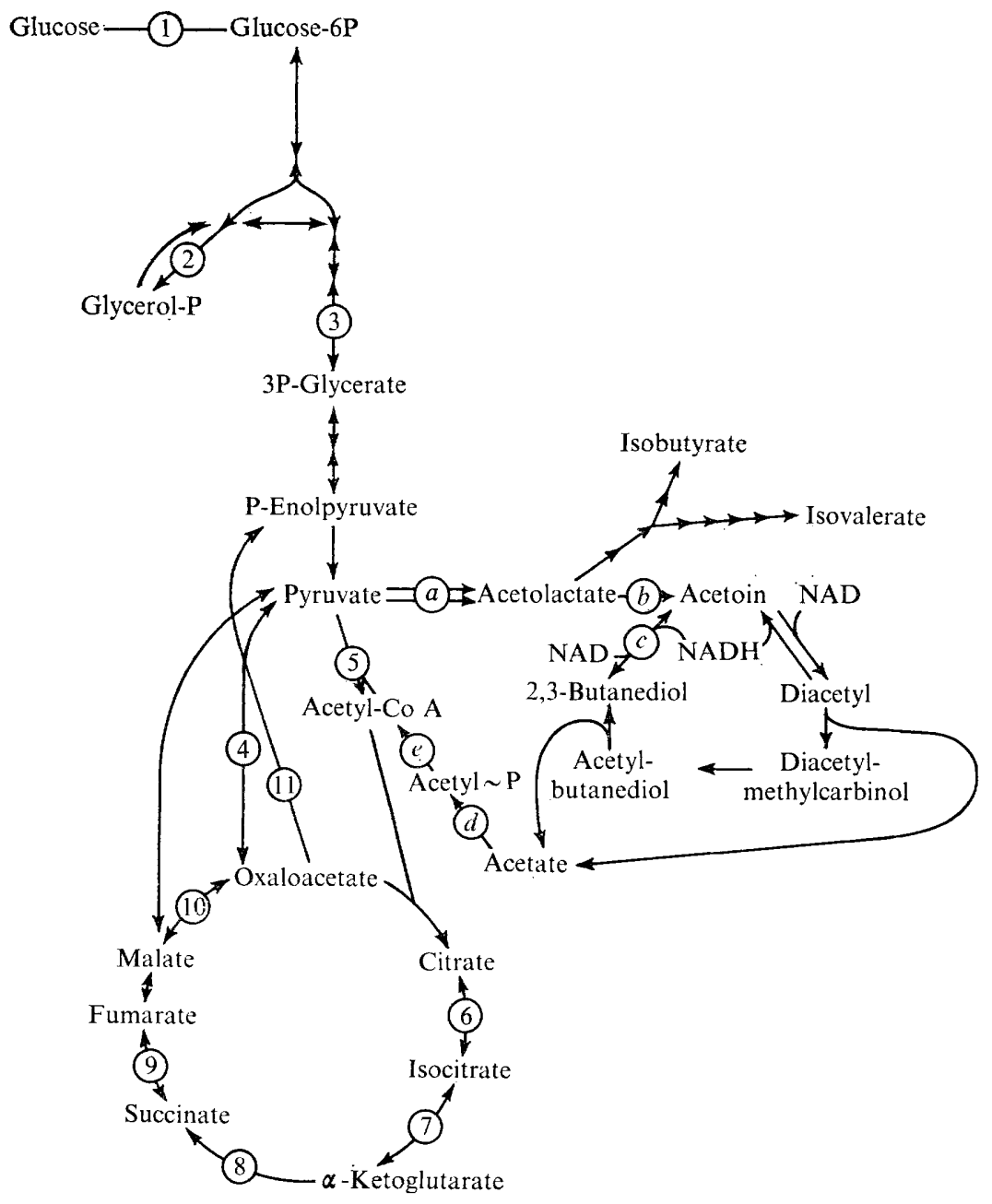

Fig. 5. Biochemical pathways of relevant metabolic conversions. The numbers mark the enzymatic reactions missing in different mutants. They are summarized in Table $\mathrm{I}$. The letters mark some of the other enzymes analysed or discussed in this paper: $(a)$ acetolactate synthase; $(b)$ acetolactate decarboxylase (EC. 4.I.I.5); (c) 2,3-butanediol dehydrogenase (EC. I.I.I.4); (d) acetate kinase (EC. 2.7.2.I); (e) phosphate acetyltransferase (EC. 2.3.1.8).

and growth virtually ceased, when air was replaced by nitrogen. Fig. 7 shows that this reaction was readily reversible; when butanediol (Io $\mathrm{mM}$ ) was added to a well-aerated culture in MG medium, more than half of it was oxidized to acetoin. In NSM+Glc, acetoin also reached its highest concentration when the culture was well aerated.

After all the glucose had been used up, the concentrations of both acetoin (Fig. 2, 4 and 8) and butanediol (Fig. 4) slowly decreased at a rate which was enhanced by aeration, indicating the oxidation of acetoin. According to Juni \& Heym (I956), this oxidation occurs via the butanediol cycle by which acetate is produced (Fig. 5). The colorimetric assay for acetoin does not differentiate between diacetyl, diacetymlethylcarbinol and acetylbutanediol. Fig. 2 implies, therefore, that acetoin and all the other colour-producing compounds taken together decreased in concentration, indicating that acetoin was slowly oxidized to acetate via the 


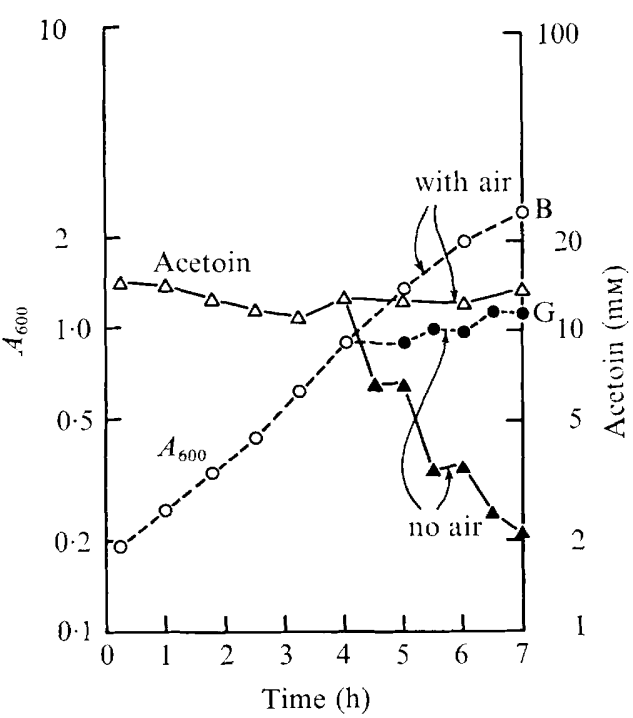

Fig. 6

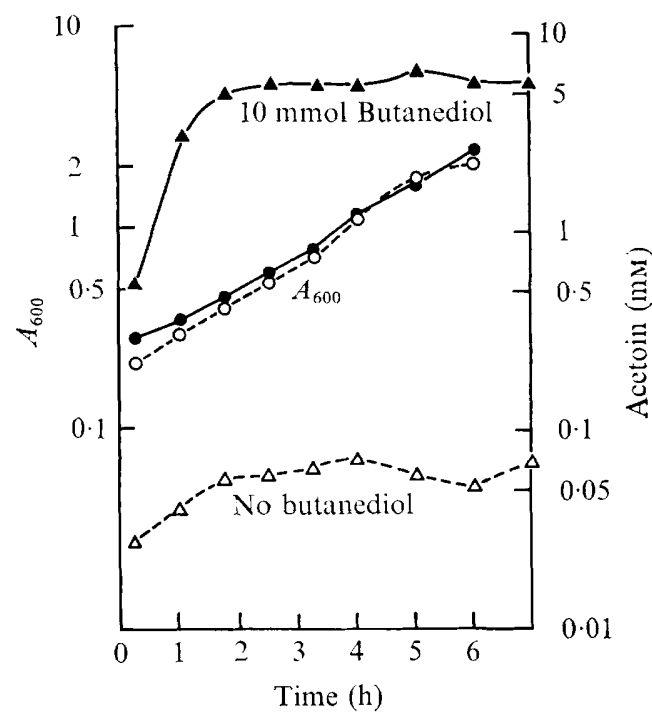

Fig. 7

Fig. 6. Reduction of acetoin in the absence of air. Strain 600I 5 was grown in minimal (M) glucose ( $1 \%$ ) medium plus $14 \mathrm{~mm}$-acetoin. After $4 \mathrm{~h}$, samples of the culture were transferred to two Erlenmeyer flasks at $37^{\circ} \mathrm{C}$, in one of which aeration was continued by shaking (open symbols), while in the other (solid symbols) air was replaced by nitrogen and the culture was no longer shaken.

Fig. 7. Oxidation of 2,3-butanediol to acetoin. Strain $600 \mathrm{I} 5$ was grown in $\mathrm{M}+\mathrm{I} \%$ Glc to an $A_{600}$ of about 0.2 and Io mm-butanediol was added to half of the culture (solid symbols), while the other half continued growth without any addition (open symbols).

butanediol cycle. This oxidation began only after glucose had been used up. Its rate increased with aeration, i.e. the efficiency of shaking.

The presence of acetoin did not significantly affect sporulation, which started after Glc had been exhausted. Even when NSM (NSMP) cultures without Glc contained $40 \mathrm{nM}$ acetoin, the frequency of sporulation after $\mathrm{I} 6 \mathrm{~h}$ was $5 \mathrm{I} \%(43 \%)$ and after $24 \mathrm{~h} 78 \%(54 \%)$, while the acetoin concentrations at these times were still $22 \mathrm{mM}$ (I $6 \mathrm{mM}$ ) and I $2 \mathrm{mM}$ (I I mM) respectively.

$p H$ changes and acetoin production in mutants. The inability of some mutants to produce or re-utilize certain metabolites was examined by measuring the decrease and later increase of the $\mathrm{pH}$, as well as the production and later metabolism of acetoin. Cultures were inoculated at an initial $A_{600}$ of $0 . \mathrm{I}$ in $25 \mathrm{~mm}$ tubes containing $5 \mathrm{ml} \mathrm{NSM}+0.5 \%$ Glc and shaken at 180 strokes/min at $37^{\circ} \mathrm{C}$. (Owing to a lower surface/volume ratio, the aeration was not quite as efficient as in the Erlenmeyer flasks used for the previous experiments.) For the standard strain 600 5 , the $\mathrm{pH}$ was lowest after 6 to $7 \mathrm{~h}$ and acetoin was at its greatest concentration after 7 to $8 \mathrm{~h}$. The $\mathrm{pH}$ then rose steadily to above $\mathrm{pH} 8$ (achieved within $24 \mathrm{~h}$ ). These conditions will be called 'normal'. The $\mathrm{pH}$ increase was absent in mutant cultures which either did not use up the glucose or which could not metabolize the product acids. The acetoin concentration normally decreased only slowly and often reached a low value only after $48 \mathrm{~h}$. The absence of acetoin metabolism could reflect the lack of complete glucose utilization, inefficient respiration, or a block in the oxidative part of the butanediol cycle.

The enzymic blocks present in some of the mutants are indicated in Fig. 5, and the findings 


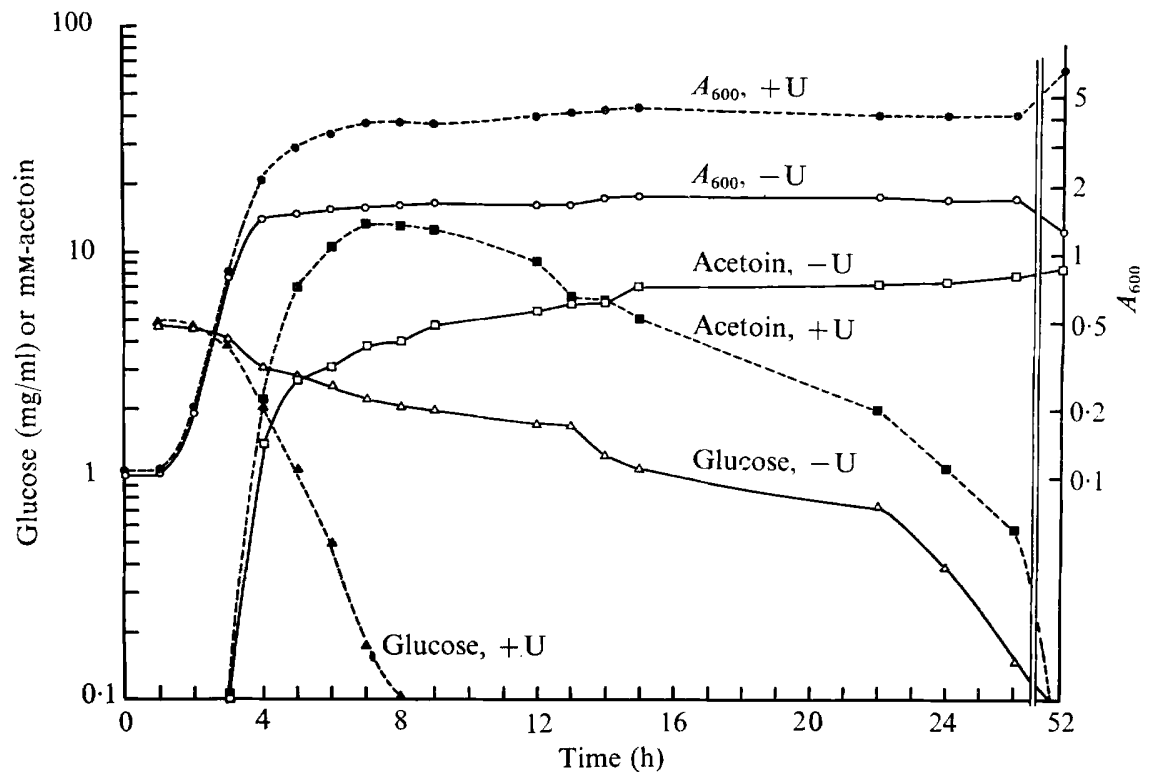

Fig. 8. Acetoin production and metabolism and glucose consumption by a uracil-requiring strain (600 I 8). The strain was grown in NSM $+0.5 \%$ Glc either with $20 \mu \mathrm{g}$ uracil $/ \mathrm{ml}(+\mathrm{U})$, or without $(-\mathrm{U})$.

are summarized in Table I. As expected, mutants unable to convert glucose into pyruvate produced only a small $\mathrm{pH}$ decrease and little acetoin.

A pyruvate carboxylase mutant (6r437), unable to grow on glucose as sole carbon source and to convert pyruvate or PEP to oxaloacetate, maintained a low $\mathrm{pH}$ and used up the produced acetoin only very slowly. Another mutant [6I I4I defective in the pyruvate dehydrogenase complex (Freese \& Fortnagel, r969)] also maintained a low pH, because it could not metabolize pyruvate to acetyl-CoA. It produced acetoin more slowly than the standard strain and was unable to re-utilize it within $48 \mathrm{~h}$, because growth stopped at an $A_{600}$ of I. 5 and glucose was metabolized only very slowly, keeping the oxidative part of the butanediol cycle repressed; thus the acetate deficiency could not be relieved by acetoin oxidation. If the medium contained ro mM-acetate the mutant grew normally and acetoin was produced and used up as in the standard strain (Table I).

All mutants lacking the activity of a citric acid cycle enzyme (Fortnagel \& Freese, 1968 a) grew to a low $A_{600}$, used up glucose very slowly, accumulated acids (low $\mathrm{pH}$ ), and produced acetoin; but they could neither increase the $\mathrm{pH}$ nor metabolize the acetoin on prolonged incubation (illustrated for a few mutants in Table I). The effect of an aconitase block could be mimicked in the standard strain by the addition of $0.5 \mathrm{mM}-\alpha$-picolinate to NSM $+0.5 \%$ Glc; $\alpha$-picolinate is known to inhibit aconitase (Fortnagel \& Freese, 1968b).

Cytochrome $a$-deficient mutants showed a response that parallels their growth properties (H. Taber, personal communication): mutants (class I, e.g. 60843) that can grow on malate (or glutamate as sole carbon source) showed the normal changes of $\mathrm{pH}$ and acetoin. But mutants unable to grow on malate (class II, 60847; class III, 60844) maintained a low pH and metabolized the produced acetoin only very slowly.

A PEP-carboxykinase mutant (6II04) showed the usual $\mathrm{pH}$ increase but did not oxidize the produced acetoin.

One hundred and four cacogenic mutants were tested whose biochemical block is unknown 


\section{Table 2. Sporulation of a uracil-requiring mutant (600 r 8)}

Bacteria were inoculated at an initial $A_{600}$ of $0 \cdot$ I into NSM medium containing the stated additions. The viable bacteria (V) and spore (S) titres were determined after $27 \mathrm{~h}$ at $37^{\circ} \mathrm{C}$.

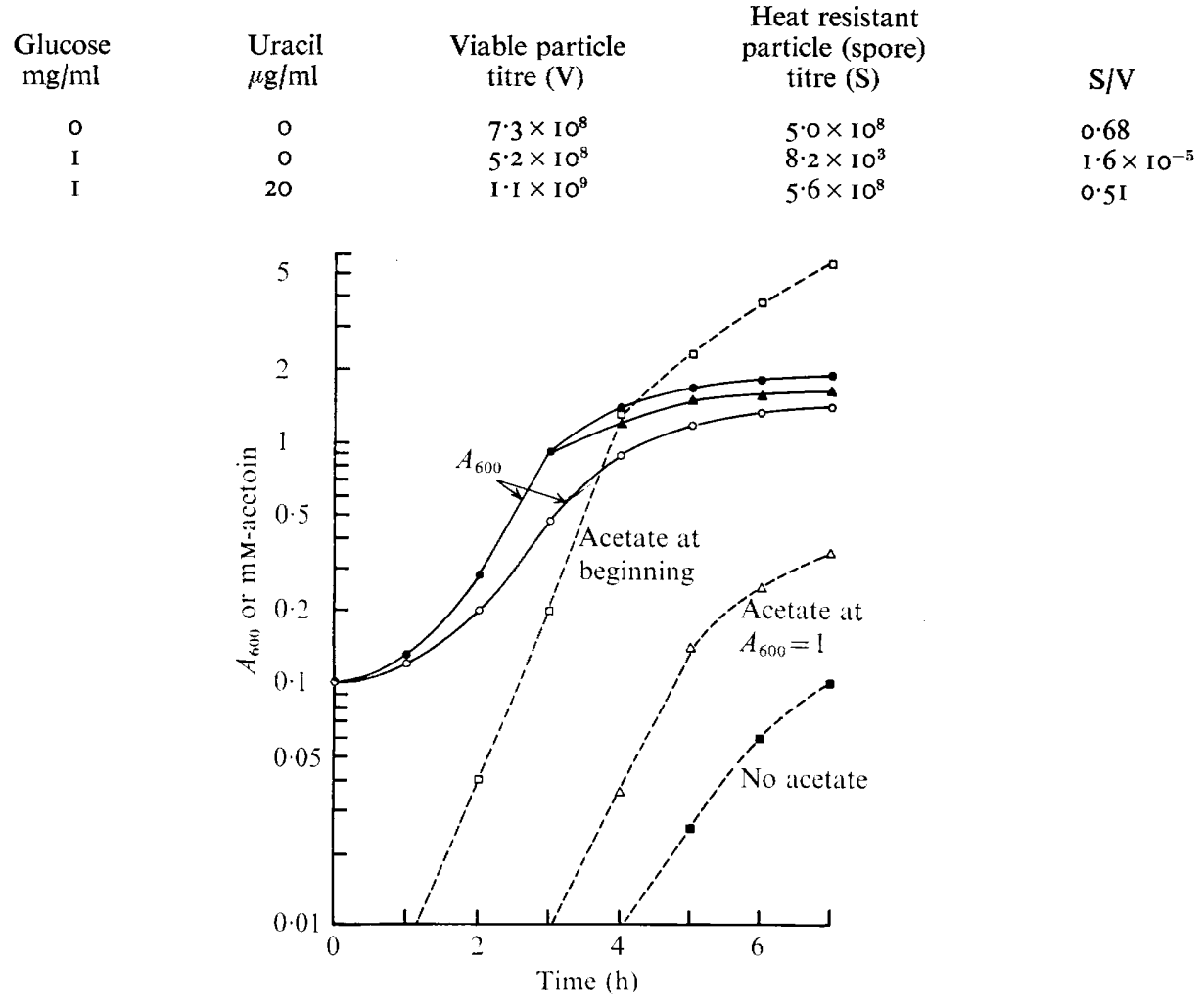

Fig. 9. Dependence of acetoin production on acetate. Strain 60015 was grown in Trp-limiting casein hydrolysate medium $\left(A_{600}, \bigcirc\right.$; acetoin, $\left.\mathbf{0}\right)$. To parts of this culture $50 \mathrm{~mm}-\mathrm{K}$-acetate $(\mathrm{pH} 7)$ were added either from the beginning $\left(A_{600}, \bigcirc\right.$; acetoin, $\square$ ), or at $A_{600}=$ I (triangles).

but which are unable to sporulate normally (Fortnagel \& Freese, I968a; Freese et al. 1969). All produced acetoin, but three strains which grew at a very slow rate (e.g. strain 60999, Table $\mathrm{r}$ ) produced it only slowly. All strains showed the usual $\mathrm{pH}$ decrease and most of them the later $\mathrm{pH}$ increase (two exceptions, 60645 and 60755, are shown in Table I). However, about $40 \%$ of the strains did not metabolize the acetoin produced fast enough to decrease its concentration below I mM in $48 \mathrm{~h}$ (whether this was because of delayed glucose utilization or some other deficiency was not determined).

A number of auxotrophs requiring known amino acids, purines or pyrimidines, vitamins or glycerol (6I106) produced the normal pH and acetoin response; NSM apparently contained sufficient amounts of the required nutrients. However, a uracil-requiring mutant (600I8) behaved exceptionally (Table I). It can be used to demonstrate the effect of glucose on acetoin production and sporulation. Table I and Fig. 8 show that without added uracil it only grew to a low $A_{600}$, did not use up the glucose for a long time, and retained both the low $\mathrm{pH}$ and the high acetoin concentration for $52 \mathrm{~h}$. But if the medium contained uracil, the strain grew to the normal $A_{600}$, used up the glucose provided, and allowed the usual $\mathrm{pH}$ rise and metabolism of acetoin. The strain sporulated normally in NSMP. But as little as 


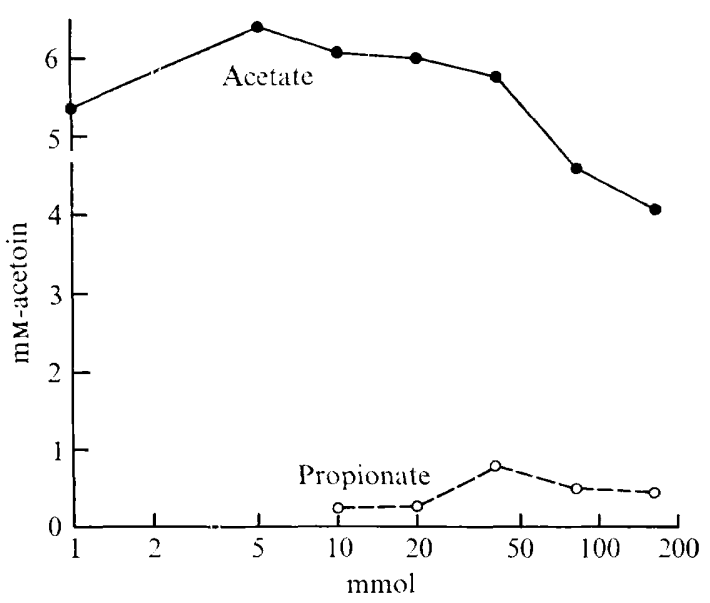

Fig. 10. Dependence of acetoin production on the presence of different compounds. Strain 600 I 5 was grown from $A_{600}=0^{\circ} \mathrm{I}$ in Trp-limiting casein hydrolysate medium containing different concentrations of various compounds (with pH adjusted to 7 where necessary). The concentration of acetoin was measured after $5 \mathrm{~h}$. Among others, the following compounds (used at $10 \mathrm{~mm}$ or at different concentrations up to slight growth inhibition) did not induce acetoin production above the base level: 2,3-butanediol, $n$-butanol, $t$-butanol, $n$-butyrate, caprate, caproate, citrate, decanoate, ethanol, ethylmalonate, formate, hexanoate, L-isoleucine, isovalerate, DL-lactate, L-leucine, linoleate, L-malate, maleate, malonate, octanoate, oxaloacetate, 2-propanol, pyruvate, L-threonine, valerate, L-valine.

O. I \% glucose sufficed to suppress sporulation (Table 2). Apparently the addition of glucose took growth to an $A_{600}$ at which uracil was exhausted and differentiation was arrested. When the exhaustion of uracil was avoided by the addition of uracil $(20 \mu \mathrm{g} / \mathrm{ml})$, normal sporulation was observed (Table 2). [0.5\% or more of glucose suppressed sporulation for $16 \mathrm{~h}$, even in the presence of uracil, because glucose could not be used up under these conditions (Freese et al. 1972).]

Control of acetoin and 2,3-butanediol production. The concentration of acetoin increased greatly when the $\mathrm{pH}$ of the culture approached its lowest value. While a comparison of buffered and unbuffered medium showed that acetoin production could not be caused by the low $\mathrm{pH}$ itself (Fig. $2 b$ ), it might be induced by one of the formed acids. Pyruvate could not be responsible, because its concentration did not change significantly at the time at which acetoin appeared (Fig. 3) and the addition of $50 \mathrm{~mm} \mathrm{~K}$-pyruvate did not advance the time of acetoin appearance. Moreover, the pyruvate dehydrogenase mutant (6II4I) grew only to $A_{600}=\mathrm{I} \cdot 5$, accumulated pyruvate, but produced acetoin later and at a lower rate than the standard strain (Freese \& Fortnagel, 1969). These properties were restored in the presence of Io mM-K-acetate, which actually caused earlier acetoin production than that observed with the standard strain grown in NSM + Glc alone.

Acetate actually induced the production of acetoin in the standard strain (Fig. 3). Since acetoin production started, without acetate addition, after the $A_{600}$ had increased beyond I, the inducing effect of acetate could be particularly well demonstrated if bacterial growth could be stopped at about $A_{600}=$ I. A particular sporulation mutant was initially used which did not produce acetoin in the absence of acetate. No amino acid, purine, pyrimidine or vitamin increased the acetoin production unless the compound enhanced growth beyond $A_{600}=$ r. Unfortunately, the mutant was lost. Reproducible conditions for the induction of acetoin production were then established by growing the standard strain in a vitamin-free 


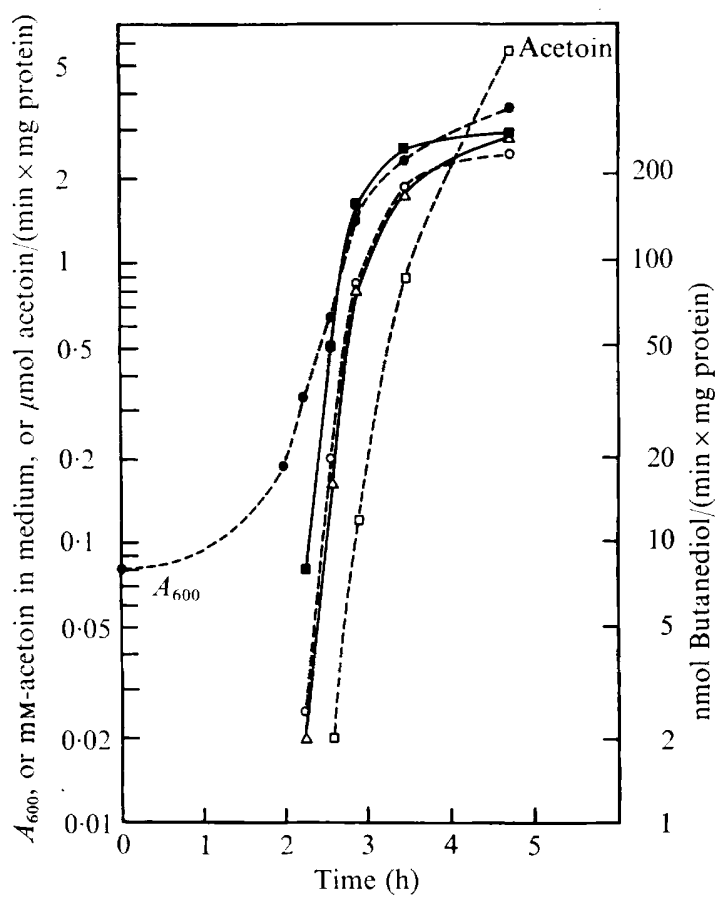

Fig. I I. Increase of butanediol-producing enzymes during growth. 600I 5 cells were grown in NSMP $+0.5 \%$ Glc, bacterial extracts were prepared at different times and the specific activities of acetolactate synthase ( $\boldsymbol{\square}$, autoclaved fraction), the 'unautoclaved fraction' $(O)$, and butanediol dehydrogenase $(\triangle)$ were assayed. In the cellular supernatant the concentration of acetoin $(\square)$ was measured.

casein hydrolysate medium to which just enough tryptophan was added to allow exponential growth to proceed to an $A_{600}$ of about I. Although the $A_{600}$ subsequently continued to rise slowly, the induction by acetate could be clearly demonstrated (Fig. 9). Acetoin production started very early if the medium initially contained $50 \mathrm{~mm}-\mathrm{K}$-acetate $(\mathrm{pH} 6 \cdot 5)$. Even if acetate was added only at the time when exponential growth stopped $\left(A_{600}=1\right)$, the concentration of acetoin still increased earlier than without acetate. The slight growth inhibition due to the high concentration $(50 \mathrm{~mm})$ of acetate was irrelevant, because maximal induction of acetoin formation was observed at an acetate concentration of about $5 \mathrm{~mm}$, which did not inhibit growth (Fig. I0). The effect of acetate was very specific, since of a large number of other compounds tested, propionate was the only one to have some inducing effect (Fig. Io).

Induction of 2,3-butanediol-forming enzymes by acetate. All three enzymes needed for the production of butanediol from pyruvate increased approximately at the same time during growth of the standard strain (600I5) in NSM $+0.5 \%$ Glc (Fig. II). (Acetolactate decarboxylase activity was initially estimated by the amount of acetoin measured before autoclaving. When acetolactate was later identified, the simultaneous induction of acetolactate synthase and decarboxylase was directly verified.) The increase of all enzyme activities was stopped by the addition of actinomycin-D $(2 \mu \mathrm{g} / \mathrm{ml})$ to the medium, indicating that mRNA synthesis was required for the de novo synthesis of the enzymes. When the NSM+Glc medium contained also $50 \mathrm{mM}$-acetate, the enzyme activities increased about $45 \mathrm{~min}$ earlier 


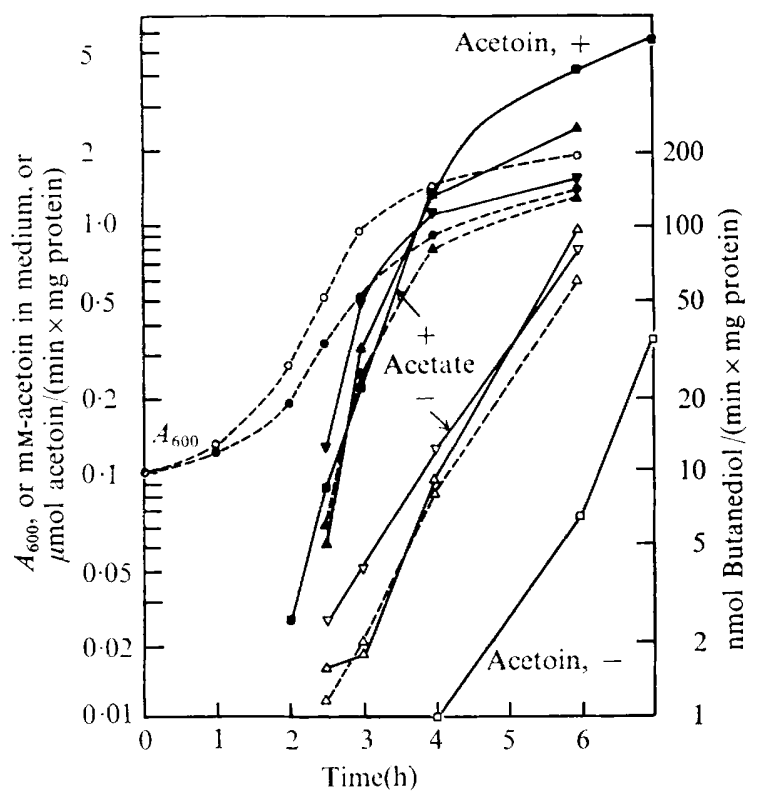

Fig. I 2. Induction of the butanediol-producing enzymes by acetate. Strain 600 5 was grown in Trplimiting casein hydrolysate medium without $(-$, open symbols) and with $(+$, solid symbols) $20 \mathrm{~mm}$ $\mathrm{K}$-acetate $\left(\mathrm{pH}_{7}\right)$. At different times cell extracts were prepared and the specific activities of acetolactate synthase $\left(\nabla-\nabla-\right.$, autoclaved), the unautoclaved fraction $\left(\triangle--\mathbf{A}_{---)}\right.$, and butanediol dehydrogenase $(\triangle-\Delta-)$ were determined. In the cellular supernatant the concentration of acetoin $(\square, \square)$ was measured.

(enzyme curves of Fig. II shifted by $45 \mathrm{~min}$ to the left), while acetoin increased about $\mathrm{I} \cdot 5 \mathrm{~h}$ earlier.

The effect of acetate on enzyme induction could be measured more effectively when the tryptophan-limiting medium was employed. As Fig. I 2 shows, all enzyme activities increased much earlier and more rapidly when the medium contained acetate. Without acetate, the production of acetoin was even more delayed than that of the enzymes. No enzyme induction was observed with Io mM-acetoin, 2,3-butanediol, L-isoleucine, or L-valine.

\section{DISCUSSION}

Following the production of pyruvate and acetate, several other metabolites are secreted during the growth of Bacillus subtilis in nutrient medium (NSM or NSMP) plus glucose; all are derived from acetolactate (Fig. 5). Large quantities of acetoin and 2,3-butanediol are followed by smaller quantities of isovalerate and isobutyrate (Fig. 4). The production of uncharged acetoin and butanediol is a useful device employed by the organism to avoid over-acidification by pyruvate or acetate. It also makes sense that pyruvate is not always converted into these compounds but that the synthesis of the necessary enzymes is induced and their function is activated only when enough acetate has been accumulated to supply the further needs of the organism. The induction of the butanediol-forming enzymes by acetate has previously been observed in Enterobacter aerogenes (Størmer, I968). Our findings in $B$. subtilis also agree with the observations in this organism that acetate activates acetolactate synthase ( $\mathrm{pH} 6$ enzyme). We have found in $B$. subtilis that the induction of the three butanediol-forming enzymes (acetolactate synthase, decarborylase and butanediol dehydrogenase) by acetate is quite specific, propionate being the only other compound with a 
small inducing effect, while, for example, other fatty acids, acetoin, 2,3-butanediol, citrate, oxaloacetate, malate, pyruvate or valine are ineffective (see Fig. 9). Since the optimal inducing concentration of acetate is $5 \mathrm{mM}$, the effect is not caused by the inhibition of growth (Sheu \& Freese, 1972) and membrane transport (Sheu, Konings \& Freese, I972), which is effective only at much higher acetate concentrations ( $50 \mathrm{~mm}$ ) and can be obtained equally well by (increasingly smaller concentrations of) longer fatty acids. It is not known whether acetate itself, acetyl-P or acetyl-CoA (or some fatty acid intermediate) is the actual inducer. In Brevibacterium flavum acetokinase is necessary for the production of acetyl-CoA from acetate (Shiio, Momose \& Oyama, I969). If that should also be the case in Bacillus subtilis, acetate itself would seem to be the inducer, because we have measured the $K_{m}$ of acetokinase (in crude extracts) with respect to acetate as $0.3 \mathrm{M}$ (and $0.5 \mathrm{M}$ with respect to propionate), which is much higher than the optimal concentration of $5 \mathrm{~mm}$-acetate required for the induction of acetoin production. In any case, acetyl-CoA (and its derivative, acetate), into which pyruvate is converted by the pyruvate dehydrogenase complex, plays a key role in controlling the metabolism of pyruvate into other compounds: they can be made only if the bacteria are assured of a sufficient supply of acetyl-CoA. This is true not only for the production of acetolactate, acetoin and butanediol, as shown here, but also for the production of oxaloacetate by pyruvate carboxylase, whose activity strictly depends on the activation by acetyl-CoA (Diesterhaft \& Freese, 1973; this paper also reports that malic enzyme is induced by growth in malate and is therefore used in the production rather than the utilization of pyruvate). This activation is not responsible for the acetoin-inducing effect of acetate, because the mutant 6I437, deficient in pyruvate carboxylase, still produces acetoin (Table I).

The interconversion of acetoin and butanediol depends on the oxidation state of the bacteria. Under reducing conditions, acetoin is converted to butanediol. Similarly, any diacetyl formed by oxidation of acetoin should be reconverted to acetoin by the diacetyl reductase which was found in Aerobacter aerogenes to be identical with butanediol dehydrogenase (Bryn, Hetland \& Størmer, 197I). This part of the butanediol cycle can therefore be used to oxidize NADH. Conversely, in good aeration (and therefore a high NAD/NADH ratio), butanediol is converted to acetoin, whose concentration (and that of all other compounds of the butanediol cycle which produce the same Voges-Proskauer colour reaction as acetoin, see Juni \& Heym, I956) decreases slowly, giving rise to acetate, but only after glucose has been used up. This agrees with the finding of Lopez \& Fortnagel (I972), who have observed that the oxidative enzymes of the butanediol cycle (acetoin dehydrogenase and diacetyl methylcarbinol synthase) are induced by acetoin but repressed by glucose. In contrast, the butanediol-forming enzymes, including butanediol dehydrogenase, which can both produce and oxidize butanediol, are not repressed by glucose.

Since Bacillus subtilis starts sporulation only after all glycolytic compounds have been metabolized, the oxidative enzymes of the butanediol cycle could be active during the developmental period. Any accumulated acetoin or butanediol might then be useful as a precursor of acetate to provide the needed acetyl-CoA for the regeneration of ATP (Freese et al. 1969).

Several mutants have been used in this paper to characterize the control of acid and acetoin production and re-utilization. Conversely, mutants of unknown biochemical deficiencies can be classified and the approximate location of their block in the metabolic pathway system can be determined according to their inability to produce or re-utilize acids or acetoin (and butanediol) in media containing different carbon sources (e.g. glucose or glycerol). For example, mutants blocked in the Embden-Meyerhof path cannot (or cannot efficiently) convert glucose to pyruvate and therefore produce only a little acetoin. Mutants blocked in 
pyruvate dehydrogenase or citric acid cycle enzymes, some mutants deficient in the electron transport system, and some sporulation mutants with unknown blocks, cannot re-utilize some of the produced acids and therefore maintain a low $\mathrm{pH}$; they can be identified on plates by the method of Carls \& Hanson (197I). While most sporulation mutants produce acetoin, many of them do not show the later decline, indicating an inability to use up glucose, a deficiency in the electron transport system, or the lack of a butanediol cycle enzyme.

\section{REFERENCES}

Bauerle, R. H., Freundlich, M., Størmer, F. C. \& Umbarger, H. E. (I964). Control of isoleucine, valine, and leucine biosynthesis. II. Endproduct inhibition by valine of acetohydroxy acid synthetase in Salmonella typhimurium. Biochimica et biophysica acta 92, I42-149.

Bryn, K., Hetland, O. \& Størmer, F. C. (1971). The reduction of diacetyl and acetoin in Aerobacter aerogenes. European Journal of Biochemistry I8, I I6-I 19.

CARLS, R. A. \& HANSON, R. S. (I97I). Isolation and characterization of tricarboxylic acid cycle mutants of Bacillus subtilis. Journal of Bacteriology 106, 848-855.

Diesterhaft, M. D. \& Freese, E. (1973). Journal of Biological Chemistry (in Press.)

Fortnagel, P. \& Freese, E. (1968a). Analysis of sporulation mutants. II. Mutants blocked in the citric acid cycle. Journal of Bacteriology 95, 1431-1438.

Fortnagel, P. \& Freese, E. ( $1968 b$ ). Inhibition of aconitase by chelation of transition metals causing inhibition of sporulation in Bacillus subtilis. Journal of Biological Chemistry 243, 5289-5295.

FreEse, E. \& ForTNAGEL, U. (1969). Growth and sporulation of Bacillus subtilis mutants blocked in the pyruvate dehydrogenase complex. Journal of Bacteriology 99, 745-756.

Freese, E., Fortnagel, P., Schmitt, R., Klofat, W., Chapelle, E. \& Picciolo, G. (1969). Biochemical genetics of initial sporulation stages. In Spores, vol. 4, pp. 82-10I. Edited by L. L. Campbell. Bethesda, Maryland: American Society for Microbiology.

Freese, E., Klofat, W. \& Galliers, E. (I970). Commitment to sporulation and induction of glucosephosphoenolpyruvate-transferase. Biochimica et biophysica acta 222, 265-289.

Freese, E., Oh, Y. K., Freese, E. B., Diesterhaft, M. D. \& Prasad, C. (1972). Suppression of sporulation of Bacillus subtilis. In Spores, vol. 5, pp. 21 2-22I. Edited by H. O. Halvorson, R. Hanson and L. L. Campbell. Washington, D.C.: American Society for Microbiology.

JUNI, E. \& HeYm, G. A. (1956). A cyclic pathway for the bacterial dissimilation of 2,3-butanediol, acetylmethylcarbinol, and diacetyl. I. General aspects of the 2,3-butanediol cycle. Journal of Bacteriology $\mathbf{7 1}$, $425-432$.

KomineK, L. A. \& HaLvorson, H. O. (1965). Metabolism of polyhydroxybutyrate and acetoin in Bacillus cereus. Journal of Bacteriology 90, 1251-1259.

KrampitZ, L. O. (1948). Synthesis of $\alpha$-acetolactic acid. Archives of Biochemistry 17, 8I-85.

LøKEN, J. P. \& STøRMER, F. C. (1970). Acetolacetate decarboxylase from Aerobacter aerogenes. Purification and properties. European Journal of Biochemistry I4, I33-I 37.

LOPEZ, J. \& ForTNAGEL, P. (1972). The regulation of the butanediol cycle in Bacillus subtilis. Biochimica et biophysica acta 279, 554-560.

Lowry, O. H., Rosebrough, N. J., Farr, A. L. \& Randall, R. J. (I95I). Protein measurement with the Folin phenol reagent. Journal of Biological Chemistry I93, 265-275.

Malthe-Sørenssen, D. \& Størmer, R. C. (1970). The pH 6 acetolacetate-forming enzyme from Serratia marcescens. Purification and properties. European Journal of Biochemistry 14, 127-1 32.

Sheu, C. W. \& Freese, E. (1972). Effects of fatty acids on growth and envelope proteins of Bacillus subtilis. Journal of Bacteriology III, 516-524.

Sheu, C. W., Konings, W. N. \& Freese, E. (1972). Effects of acetate and other short-chain fatty acids on sugar and amino acid uptake of Bacillus subtilis. Journal of Bacteriology III, 525-530.

Shiro, I., Momose, H. \& Oyama, A. (I969). Genetic and biochemical studies on bacterial formation of L-glutamate. Journal of General and Applied Microbiology 15, 27-40.

Smith, N. R., Gordon, R. E. \& Clark, F. E. (1952). Aerobic Sporeforming Bacteria. Agriculture Monograph Number I6. Washington: United States Department of Agriculture.

STøRMER, F. C. (1968). Evidence for induction of the 2,3-butanediol-forming enzymes in Aerobacter aerogenes. Federation of European Biochemical Societies Letters 2, 36-38.

Yoshida, A. \& Freese, E. (1965). Purification and chemical characterization of lactate dehydrogenase of Bacillus subtilis. Biochimica et biophysica acta 99, 56-65. 